\title{
Arterial Blood Gas as a Predictor of Mortality in COVID Pneumonia Patients Initiated on Noninvasive Mechanical Ventilation: A Retrospective Analysis
}

\author{
Bhavna Gupta ${ }^{1}$, Gaurav Jain $^{2} \odot$, Saurabh Chandrakar $^{3} \odot$, Nidhi Gupta ${ }^{4}$, Ankit Agarwal ${ }^{5} \odot$
}

\begin{abstract}
Background:The alveolar-arterial oxygen $(\mathrm{A}-\mathrm{a})$ gradient measures the difference between the oxygen concentration in alveoli and the arterial system, which has considerable clinical utility.

Materials and methods: It was a retrospective, observational cohort study involving the analysis of patients diagnosed with acute COVID pneumonia and required noninvasive mechanical ventilation (NIV) over a period of 3 months. The primary objective was to investigate the utility of the A-a gradient (pre-NIV) as a predictor of 28-day mortality in COVID pneumonia. The secondary objective included the utility of other arterial blood gas (ABG) parameters (pre-NIV) as a predictor of 28-day mortality. The outcome was also compared between survivors and nonsurvivors. The outcome variables were analyzed by receiver-operating characteristic (ROC) curve, Youden index, and regression analysis.

Results: The optimal criterion for A-a gradient to predict 28-day mortality was calculated as $\leq 430.43$ at a Youden index of 0.5029 , with the highest area under the curve (AUC) of $0.755(p<0.0001)$. On regression analysis, the odds ratio for the A-a gradient was 0.99 . A significant difference was observed in $\mathrm{ABG}$ predictors, including $\mathrm{PaO}_{2}, \mathrm{PaCO}_{2}, \mathrm{~A}-\mathrm{a}$ gradient, $\mathrm{AO}_{2}$, and arterial-alveolar $(\mathrm{a}-\mathrm{A})(\%)$ among nonsurvivors vs survivors ( $p$-value $<0.001)$. The vasopressor requirement, need for renal replacement therapy, total parenteral requirement, and blood transfusion were higher among nonsurvivors; however, a significant difference was achieved with the vasopressor need $(p<0.001)$.

Conclusion: This study demonstrated that the A-a gradient is a significant predictor of mortality in patients initiated on NIV for worsening respiratory distress in COVID pneumonia. All other ABG parameters also showed a significant AUC for predicting 28-day mortality, although with variable sensitivity and specificity.

Key messages: COVID-19 pneumonia shows an initial presentation with type 1 respiratory failure with increased A-a gradient, while a subsequent impending type 2 respiratory failure requires invasive ventilation.

A significant difference was observed in $\mathrm{ABG}$ predictors, including $\mathrm{PaO}_{2}, \mathrm{PaCO}_{2}, \mathrm{~A}$-a gradient, $\mathrm{AO}_{2}$, and a-A (\%) among nonsurvivors vs survivors. ( $p$-value $<0.001)$.

The vasopressor requirement, need for renal replacement therapy, total parenteral requirement, and blood transfusion need were higher among nonsurvivors than survivors; however, a significant difference was achieved with the vasopressor need $(p<0.001)$.

Keywords: COVID-19 Acute Respiratory Distress Syndrome, Critically ill adults, Mortality predictors.

Indian Journal of Critical Care Medicine (2021): 10.5005/jp-journals-10071-23917
\end{abstract}

\section{INTRODUCTION}

Severe acute respiratory syndrome-related coronavirus-2 (SARS-CoV-2) is a virus-targeted respiratory disorder with a broad range of symptoms ranging from asymptomatic cases to severe pneumonia or acute respiratory distress syndrome $(A R D S)^{1}$; the reported mortality rate is as high as $78 \%$ in critically ill patients. ${ }^{2}$ For efficient use of limited healthcare resources, early detection of risk factors and timely management are critical to minimize the adverse outcome. ${ }^{3}$

The alveolar-arterial oxygen gradient ( $A$-a gradient), which measures the difference between the oxygen concentration in alveoli and the arterial system, has considerable clinical utility in narrowing the differential diagnosis for the cause of hypoxemia. ${ }^{4}$ Although a perfect system should have a zero A-a gradient, the underlying heterogeneity in capillary perfusion and alveolar ventilation from the apical to basal lung units leads to a physiological A-a gradient throughout the lung. It is further affected by demographic characteristics and various pathological
${ }^{1-5}$ Department of Anaesthesia and Critical Care, All India Institute of Medical Sciences, Rishikesh, Uttarakhand, India

Corresponding Author: Nidhi Gupta, Department of Anaesthesia and Critical Care, All India Institute of Medical Sciences, Rishikesh, Uttarakhand, India, Phone: +91 9760672721, e-mail:nidhigupta.doon @gmail.com

How to cite this article: Gupta B, Jain G, Chandrakar S, Gupta N, Agarwal A. Arterial Blood Gas as a Predictor of Mortality in COVID Pneumonia Patients Initiated on Noninvasive Mechanical Ventilation: A Retrospective Analysis. Indian J Crit Care Med 2021;25(8):866-871.

Source of support: Nil

Conflict of interest: None

processes. The pulmonary disorders that alter the ventilationperfusion ratio or disturb the oxygen transfer from the alveoli to pulmonary circulation compromise the arterial oxygenation and increase this gradient.

(0) Jaypee Brothers Medical Publishers. 2021 Open Access This article is distributed under the terms of the Creative Commons Attribution 4.0 International License (https://creativecommons.org/licenses/by-nc/4.0/), which permits unrestricted use, distribution, and non-commercial reproduction in any medium, provided you give appropriate credit to the original author(s) and the source, provide a link to the Creative Commons license, and indicate if changes were made. The Creative Commons Public Domain Dedication waiver (http://creativecommons.org/publicdomain/zero/1.0/) applies to the data made available in this article, unless otherwise stated. 
The recent literature on COVID-19 pneumonia shows an initial presentation with type 1 respiratory failure with increased A-a gradient, while a subsequent impending type 2 respiratory failure requires invasive ventilation. ${ }^{5} \mathrm{~A}$ higher $\mathrm{A}$-a gradient at the initiation of noninvasive mechanical ventilation (NIV) signifies a higher degree of ventilation-perfusion mismatch or interstitial pathology. So, we hypothesized that it should reflect subsequent mortality in such patients. Thus, we aimed to assess the utility of ABG parameters as a predictor of 28-day mortality in COVID pneumonia patients initiated on NIV.

\section{Materials and Methods}

After institutional ethical clearance (via letter no AIIMS/ IEC/21/17, dated January 9, 2021), under a retrospective, observational cohort design, this study involved the collection, classification, and analysis of patients aged $>18$ years of either sex, admitted to this tertiary care institute in between July and September 2020 (3-month data), diagnosed with acute COVID pneumonia, and required NIV. We excluded those with any previous history of cardiopulmonary disease, renal disorder, psychiatric illness, pregnancy, lactation, recent hospitalization (past 3 months), immunocompromised status, or malignancy. We also followed the patient's data till discharge or death or 28 days since admission, whichever occurred first, through hospital records.

We categorized a patient as COVID-positive case on getting a positive reverse-transcriptase-polymerase chain reaction report from a nasal or pharyngeal swab specimen. According to the institution guidelines, patients were classified as moderateor severe based on the presence of hypoxia $\left(\mathrm{SpO}_{2}<93 \%\right)$ or radiological evidence of pneumonia or ARDS and organ impairment and shock. A high-resolution computed tomographic scan was done for COVID-19 patients with inconclusive chest $\mathrm{X}$-ray and persistent symptoms. The indication for initiation of NIV included standard protocol as followed in our tertiary care institute. The indications included symptoms of ARDS, such as dyspnea at rest, respiratory rate $>25$ breath/minute, use of accessory respiratory muscles, or paradoxical breathing, and signs of disturbed alveolar gas exchange in $\mathrm{ABG}$ parameters, including partial pressure of carbon dioxide $\left(\mathrm{PaCO}_{2}\right)>45 \mathrm{~mm} \mathrm{Hg}$, arterial power of hydrogen $(\mathrm{pH})<7.25$, the partial pressure of oxygen $\left(\mathrm{PaO}_{2}\right)<60 \mathrm{~mm} \mathrm{Hg}$ or ratio of the partial pressure of oxygen or fraction of inspired oxygen $\left(\mathrm{PaO}_{2} / \mathrm{FiO}_{2}\right)<200$. The indication for initiation of invasive mechanical ventilation included the following: poor patient compliance/leaking interface with no improvement in respiratory condition despite all efforts, Glasgow coma scale $<9$ or deterioration $>3$, hemodynamic instability, cardiac arrhythmia, the inability of the NIV to improve respiratory distress or gas exchange disturbance within 2 hours of initiation, apnea or respiratory rate $(\mathrm{RR})>35$ breaths/minute, or inability to maintain a patent airway.

The data collected comprised stay and 28-day mortality. The ABG parameters included $\mathrm{PaO}_{2}, \mathrm{PaCO}_{2}$, bicarbonate $\left(\mathrm{HCO}_{3}\right)$, a fraction of inspired oxygen concentration $\left(\mathrm{FiO}_{2}\right), \mathrm{PaO}_{2} / \mathrm{FiO}_{2}, \mathrm{~A}-\mathrm{a}$ gradient, respiratory index, and arterial-alveolar (a-A) oxygen ratio, by following equations:

- A-a ratio was calculated by the following equations:

$\mathrm{PaO}_{2}=\mathrm{FiO}_{2}\left(\mathrm{P}_{\mathrm{B}}-47\right)-\mathrm{PaCO}_{2} / \mathrm{R}$

$\mathrm{PaO}_{2}=0.21 \times(760-47)-\mathrm{PaCO}_{2} / 0.8$
$\mathrm{PaO}_{2}=150-\mathrm{PaCO}_{2} / 0.8$

Here $\mathrm{PaO}_{2}=$ partial pressure of alveolar oxygen, $\mathrm{PaCO}_{2}=$ partial pressure of arterial carbon dioxide, $\mathrm{FiO}_{2}=$ fraction of inspired oxygen, Patm = atmospheric pressure (at sea level $=760 \mathrm{~mm} \mathrm{Hg}$ ), $\mathrm{PH}_{2} \mathrm{O}=$ water partial pressure in alveolus, $100 \%$ saturated (at sea level $=47 \mathrm{~mm} \mathrm{Hg}$ ) and $\mathrm{R}=$ respiratory quotient (normally 0.8).

- Respiratory index was calculated by dividing $\mathrm{pO}_{2}$ from A-a gradient:

Respiratory index $(\mathrm{RI})\left(\mathrm{RI}=\mathrm{pO}_{2}(\mathrm{~A}-\mathrm{a}) / \mathrm{pO}_{2}(\mathrm{a})\right)$

- $\mathrm{PaO}_{2} / \mathrm{FiO}_{2}$ ratio was calculated by dividing alveolar oxygen to inspired oxygen

- a-A ratio was calculated as a ratio of arterial and alveolar $\mathrm{PaO}_{2}$.

\section{Aims and Objectives}

The primary objective was to investigate the utility of A-a gradient (pre-NIV) as a predictor of 28-day mortality in COVID pneumonia patients initiated on NIV.

The secondary objective included the utility of other ABG parameters (pre-NIV) as a predictor of 28-day mortality in the above patients. The outcome studied in terms of ABG parameters and other variables like the vasopressor requirement, the need for renal replacement therapy, total parenteral requirement, and blood transfusion was compared between survivors and nonsurvivors.

The sample size was calculated using Medcalc statistical software version 14.8.1. Taking into account a receiver-operating characteristic (ROC) curve value of 0.70 as significant for pre-NIV A-a gradient as a predictor of 28-day mortality, a null hypothesis ROC curve value of 0.50 , an alpha error of 0.05 , an $80 \%$ power, and a survival: mortality ratio of $3: 1$, we required sample size of 88 (66:22). To increase the significance of results, we aim to recruit all eligible cases for the assessment period. The statistical analysis was performed with Statistical Package for the Social Sciences version 23.0 software (SPSS, IBM Corp. Armonk, New York, United States). The data were expressed as means and standard deviation (SD) or as number and percentage. The outcome variables were analyzed by a ROC curve, Youden index, and regression analysis. Continuous data were analyzed by Student's $t$-test. The Chi-square test analyzed the categorical parameters. A $p$-value $<0.05$ denotes statistical significance.

\section{Results}

We included 165 patients in the final analysis. Thirty-four patients expired (nonsurvivors), while the remaining (survivors) had a successful outcome. The mean age of included patients was 53.3 years; males were $61 \%$ and females $39 \%$, most had no comorbidities, while others had hypertension or diabetes. The mean pre-NIV A-a gradient was 403.83 (range: $379.66-427.99$ ). The mean $\mathrm{FiO}_{2}$ patients were receiving at this time point was $69.7 \%$ (range: $21-100 \%$ ) through nasal prongs, face masks, venturi masks, or rebreathing masks. The mean pre-NIV $\mathrm{PaO}_{2}$ was $56.03 \mathrm{~mm} \mathrm{Hg}$ (range: 28-96 mm Hg), and the mean $\mathrm{PaCO}_{2}$ was $29.7 \mathrm{~mm} \mathrm{Hg}$ (range: $18-53 \mathrm{~mm} \mathrm{Hg}$ ), suggesting type I respiratory failure in the majority of the patients. The mean $\mathrm{pH}$ was 7.432 (range 7.28-7.54), while the mean $\mathrm{HCO}_{3}$ was $21.48 \mathrm{mEq} / \mathrm{L}$ (range 12.5-34), and the mean $\mathrm{pO}_{2} / \mathrm{FiO}_{2}$ ratio was 89.87 (22.3-217.85) (Table 1). Mean ICU stay was 14.2 days. 
For validity analysis, we plotted a ROC curve for each ABG parameter. The optimal criterion for $\mathrm{A}-\mathrm{a}$ gradient to predict 28-day mortality was calculated as $\leq 430.43$ (sensitivity: $67.94 \%$, specificity: $82.35 \%$ ) at a Youden index of 0.5029 , with the highest area under the curve (AUC) of 0.755 ( $p<0.0001$ ). On regression analysis (Table 2), the odds ratio (OR) for the A-a gradient was 0.99 , indicating no further increase in the log-odds of mortality with an increase in $\mathrm{A}$-a gradient above the threshold value.

The optimal criterion for $\mathrm{PaO}_{2} / \mathrm{FiO}_{2}$ to predict 28-day mortality was measured as $>110$ (sensitivity $31.3 \%$, specificity $100 \%$ ) at a Youden index of 0.31 and AUC of 58\% ( $p$-value: 0.0793 ). The optimal criterion for the respiratory index to predict 28-day mortality was found to be $\leq 4.96$ (sensitivity $35.11 \%$, specificity $100 \%$ ) at a Youden index of 0.35 and AUC of $60 \%$ ( $p$-value: 0.0194$)$. The optimal criterion for a-A percentage to predict 28-day mortality was observed to be $>24.9$ (sensitivity $35 \%$, specificity $100 \%$ ) at a Youden index of 0.35 and AUC of $60 \%$ ( $p$-value: 0.0194 ) The optimal criterion for $\mathrm{PaCO}_{2}$ to predict 28-day mortality was observed to be $>24.9$ (sensitivity $88.5 \%$, specificity $55.9 \%$ ) at a

Table 1: Pre-noninvasive ventilation, arterial blood gas, and other parameters

\begin{tabular}{lcc}
\hline Parameters & Mean and SD & $95 \% \mathrm{Cl}$ \\
\hline Age (years) & $53.30 \pm 12.99$ & $51.305-55.301$ \\
ICU length of stay (days) & $14.20 \pm 6.80$ & $4-40$ \\
A-a gradient & $403.83 \pm 157.21$ & $379.663-427.998$ \\
$\mathrm{PaO}_{2} / \mathrm{FiO}_{2}$ & $89.88 \pm 3.42$ & $22.3-217.86$ \\
$\mathrm{Respiratory}$ index & $8.35 \pm 0.40$ & $1.39-28.99$ \\
$\mathrm{~A}-\mathrm{A}(\%)$ & $26.08 \pm 2.75$ & $3.58-250$ \\
$\mathrm{FiO}_{2}$ & $69.70 \pm 22.02$ & $66.318-73.088$ \\
$\mathrm{HCO}_{3}$ & $21.48 \pm 12.98$ & $19.489-23.482$ \\
$\mathrm{~K}^{+}$ & $3.74 \pm 0.48$ & $3.672-3.823$ \\
$\mathrm{Na}^{+}$ & $135.07 \pm 6.46$ & $134.085-136.073$ \\
$\mathrm{PaCO}_{2}$ & $29.692 \pm 6.08$ & $28.756-30.627$ \\
$\mathrm{PaO}_{2}$ & $56.03 \pm 19.54$ & $53.033-59.042$ \\
$\mathrm{PaO}_{2} / \mathrm{FiO}_{2}$ & $89.87 \pm 43.94$ & $83.119-96.632$ \\
$\mathrm{pH}^{\mathrm{PaCO}}$ & $7.432 \pm 0.06$ & $7.423-7.442$ \\
\hline
\end{tabular}

$\mathrm{PaCO}_{2}$, partial pressure of carbon dioxide $(\mathrm{mm} \mathrm{Hg}) ; \mathrm{pH}$, arterial power of hydrogen; $\mathrm{PaO}_{2}$, the partial pressure of oxygen $(\mathrm{mm} \mathrm{Hg}) ; \mathrm{PaO}_{2} / \mathrm{FiO}_{2}$, ratio of the partial pressure of oxygen or fraction of inspired oxygen; $\mathrm{A}$-a gradient, alveolar-arterial gradient $(\mathrm{mm} \mathrm{Hg}) ; \mathrm{HCO}_{3}$, bicarbonate $(\mathrm{mEq} / \mathrm{L}) ; \mathrm{a}-\mathrm{A}(\%)$, arterial-alveolar (percentage); $\mathrm{K}^{+}$, Potassium (mEq/L); $\mathrm{Na}^{+}$, Sodium (mEq/L); $\mathrm{SD}$, standard deviation; $\mathrm{Cl}$, confidence interval
Youden index of 0.388 and AUC of $72.9 \%$ ( $p$-value $<0.0001$ ) The optimal criterion of $\mathrm{PaO}_{2}$ to predict 28-day mortality was observed to be $\leq 54$ (sensitivity $56.5 \%$, specificity: $82.5 \%, \% L R+3.2$ ) at a Youden index of 0.44 and AUC of $66.7 \%$ ( $p$-value: 0.0014$)$. The optimal $\mathrm{pH}$ criterion to predict 28-day mortality was observed to be $\leq 7.468$ (sensitivity $79.4 \%$, specificity: $64.7 \%, \% \mathrm{LR}+2.25$, LR-0.32) at a Youden index of 0.44 and AUC of $66 \%$ ( $p$-value: 0.0076). The optimal criterion of $\mathrm{HCO}_{3}$ to predict 28-day mortality was observed to be $>19.7$ (sensitivity: $61.1 \%$, specificity: $82.4 \%$ ) at a Youden index of 0.4342 and an AUC of $66.3 \%$ ( $p$-value: 0.0016 ) (Table 3 and Fig. 1).

A significant difference was observed in $A B G$ predictors, including $\mathrm{PaO}_{2}, \mathrm{PaCO}_{2}, \mathrm{~A}-\mathrm{a}$ gradient, $\mathrm{AO}_{2}$, and a-A (\%) among nonsurvivors vs survivors ( $p$-value $<0.001)$. The vasopressor requirement, need for renal replacement therapy, total parenteral requirement, and blood transfusion need were higher among nonsurvivors than survivors; however, a significant difference was achieved with the vasopressor need ( $p<0.001$ ) (Table 4).

\section{Discussion}

This study demonstrated that the A-a gradient is a significant predictor of mortality in patients initiated on NIV for worsening respiratory distress in COVID pneumonia. The optimal criterion for the A-a gradient to predict above was observed to be $\leq 430.43$. All other ABG parameters also showed a significant AUC for predicting 28-day mortality, although with variable sensitivity and specificity.

It is known that increased cardiopulmonary vascular shunt or altered alveolar diffusion barrier can substantially affect the A-a gradient. ${ }^{6}$ Given the impact of COVID-19 interstitial pulmonary involvement on gas exchange, ventilation-perfusion mismatch, ${ }^{7}$ and shunting, we decided to test ABG parameters' validity, particularly A-a gradient, in predicting mortality. The available data indicate that around 40\% of COVID-19 patients develop ARDS (20\% severe cases), with $5-10 \%$ requiring ICU admission and invasive ventilation. Although most patients survive an acute illness, a subset develops fibro-proliferative response marked

Table 3: Regression analysis for A-a gradient

\begin{tabular}{lclc}
\hline Variable & Odds ratio & $95 \% \mathrm{Cl}$ & $p$-value \\
\hline A-a gradient & 0.9934 & $0.9904-0.9964$ & $<0.0001$ \\
Sample size & 165 & Nonsurvivors: 34 (20.61\%) \\
& & Survivors: 131 (79.39\%) \\
\hline
\end{tabular}

A-a gradient, alveolar-arterial gradient $(\mathrm{mm} \mathrm{Hg}) ; \mathrm{Cl}$, confidence interval

Table 2: Validity parameters for arterial blood gas and derived parameters

\begin{tabular}{lllllrrrr}
\hline Parameter & $A U C$ & $Y I$ & Criterion & Sensitivity & \multicolumn{1}{c}{ Specificity } & $+L R$ & $-L R$ & $p$-value \\
\hline A-a gradient & 0.76 & 0.5 & $\leq 430.43$ & $67.94(59.2-75.8)$ & $82.35(65.5-93.2)$ & 3.85 & 0.39 & $<0.0001$ \\
$\mathrm{PaO}_{2} / \mathrm{FiO}_{2}$ & 0.58 & 0.31 & $>110$ & $31.30(23.5-40.0)$ & $100.00(89.7-100.0)$ & 0.69 & 0.0793 \\
Respiratory index & 0.60 & 0.35 & $\leq 4.96$ & $35.11(27.0-43.9)$ & $100(89.7-100.0)$ & 0.0194 \\
$\mathrm{~A}-\mathrm{A}(\%)$ & 0.60 & 0.35 & $>24.99$ & $35.11(27.0-43.9$ & $100.00(89.7-100.0)$ & 0.65 & 0.0194 & 0.0194 \\
$\mathrm{PaO}_{2}$ & 0.67 & 0.39 & $\leq 54$ & $56.49(47.6-65.1)$ & $82.35(65.5-93.2)$ & 3.2 & 0.53 & 0.0014 \\
$\mathrm{PaCO}_{2}$ & 0.73 & 0.44 & $>24.9$ & $88.55(81.8-93.4)$ & $55.88(37.9-72.8)$ & 2.01 & 0.2 & $<0.0001$ \\
$\mathrm{pH}$ & 0.66 & 0.44 & $\leq 7.47$ & $79.39(71.4-86.0)$ & $64.71(46.5-80.3)$ & 2.25 & 0.32 & 0.0076 \\
$\mathrm{HCO}_{3}$ & 0.66 & 0.43 & $>19.7$ & $61.07(52.2-69.5)$ & $82.35(65.5-93.2)$ & 3.46 & 0.47 & 0.0016 \\
\hline
\end{tabular}

$\mathrm{PaCO}_{2}$, carbon dioxide; $\mathrm{pH}$, arterial power of hydrogen; $\mathrm{PaO}_{2}$, the partial pressure of oxygen; $\mathrm{PaO}_{2} / \mathrm{FiO}_{2}$, ratio of the partial pressure of oxygen or fraction of inspired oxygen; A-a gradient, alveolar-arterial gradient; $\mathrm{HCO}_{3}$, bicarbonate; a-A (\%), arterial-alveolar (percentage) 
ABG as a Predictor of Mortality in COVID Pneumonia Patients on NIV

Table 4: Comparison of demographic, arterial blood gas, and other outcome parameters among survivors vs nonsurvivors

\begin{tabular}{lccc}
\hline Parameters & Survivors $(n=131)$ & Nonsurvivors $(n=34)$ & $p$-value \\
\hline Age & $51.34 \pm 13.09$ & $60.97 \pm 9.34$ & 0.154 \\
$\mathrm{pH}$ & $7.43 \pm 0.0595$ & $7.46 \pm 0.061$ & 0.012 \\
$\mathrm{pCO}_{2}(\mathrm{~mm} \mathrm{Hg})$ & $30.67 \pm 6.08$ & $25.93 \pm 4.49$ & 0.000 \\
$\mathrm{pO}_{2}(\mathrm{~mm} \mathrm{Hg})$ & $53.77 \pm 19.29$ & $64.79 \pm 18.24$ & 0.003 \\
$\mathrm{HCO}_{3}(\mathrm{~mm} \mathrm{Hg})$ & $22.21 \pm 14.37$ & $18.70 \pm 3.7$ & 0.162 \\
$\mathrm{~A}-\mathrm{a}$ gradient based on $\mathrm{pO}_{2}$ & $375.49 \pm 157.15$ & $513.04 \pm 100.31$ & 0.000 \\
$\mathrm{ICU}$ length of stay & $13.927 \pm 6.86$ & $15.206 \pm 6.61$ & 0.334 \\
respiratory index & $8.27 \pm 5.6$ & $8.69 \pm 3.22$ & 0.676 \\
$\mathrm{AO}_{2}$ & $321.71 \pm 161.2$ & $448.25 \pm 99.92$ & 0.000 \\
$\mathrm{a}-\mathrm{A}$ (\%) & $28.90 \pm 39.21$ & $15.19 \pm 5.75$ & 0.000 \\
Mechanical ventilation days & $12.00 \pm 1.2$ & $11.40 \pm 5.56$ & 0.917 \\
Vasopressor requirement & $0.130 \pm 0.33$ & $0.515 \pm 0.50$ & 0.000 \\
$\mathrm{CRRT}$ requirement & $0.137 \pm 0.345$ & $0.176 \pm 0.387$ & 0.568 \\
TPN requirement & $0.092 \pm 0.28$ & $0.176 \pm 0.38$ & 0.159 \\
Blood transfusion requirement & $0.099 \pm 0.30$ & $0.206 \pm 0.410$ & 0.09 \\
\hline
\end{tabular}

$\mathrm{PaCO}_{2}$, carbon dioxide; $\mathrm{pH}$, arterial power of hydrogen; $\mathrm{PaO}_{2}$, the partial pressure of oxygen; $\mathrm{PaO}_{2} / \mathrm{FiO}_{2}$, ratio of the partial pressure of oxygen to fraction of inspired oxygen; $\mathrm{A}-\mathrm{a}$ gradient, alveolar-arterial gradient; $\mathrm{HCO}_{3}$, bicarbonate; a-A (\%), arterialalveolar (percentage); CRRT, continuous renal replacement therapy; TPN, total parenteral nutrition

by fibroblast aggregation and deposition of collagen and other extracellular matrix components in the lung. Historically, the occurrence of severe fibro-proliferative pulmonary ${ }^{8}$ disease has been associated with a poor prognosis, causing high mortality and/or prolonged ventilation dependence. In the present study, we found that the A-a gradient effectively predicted mortality in moderate to severe COVID-19 patients. Previous studies ${ }^{9}$ have shown that the A-a gradient was higher among nonsurvivors than survivors who had community-acquired pneumonia (AUC 0.78), with a mean A-a gradient of 148.64 and 90.16 among nonsurvivors and survivors, respectively. In another published literature, $\mathrm{AaDO}_{2}$ (A-a gradient) and $\mathrm{AaDO}_{2}$ augmentation displayed good accuracy (AUC: 0.952 and 0.810 , respectively) to predict ICU admission in patients with COVID-19. However, their cutoff value was $56.6 \pm 17.5$ in the ICU group and $25.9 \pm 9.7$ in the non-ICU group; given small sample size, strong emphasis cannot be laid upon results. Farina et al. ${ }^{10}$ also investigated the role of $\mathrm{A}$-a gradient in predicting the need for hospitalization, the survival rate, and identifying pneumonia in patients with SARS-CoV-2 infection. They reported that out of 168 patients with $\mathrm{AaDO}_{2} \leq 27$, only $3(1.8 \%)$ required readmission within 7 days; it could be attributed to the inclusion of patients with less disease severity. In our study, all patients were in respiratory failure requiring NIV, which could account for higher cutoff values of observed mean $\mathrm{A}$-a gradient.

In recent years, the a-A tension ratio $\left(\mathrm{PaO}_{2} / \mathrm{PaO}_{2}\right)$, sometimes denoted $\mathrm{a}-\mathrm{APO}_{2}$, has been used as an index of pulmonary gas exchange. While the $\mathrm{A}-\mathrm{a}$ tension difference $\left(\mathrm{A}-\mathrm{aPO}_{2}\right)$ is known to depend strongly on the $\mathrm{FiO}_{2}, \mathrm{a}-\mathrm{APO}_{2}$ is less dependent on the extrapulmonary factors. ${ }^{11}$ The $\mathrm{PaO}_{2} / \mathrm{FiO}_{2}$ ratio is also widely used as a clinical indicator of hypoxemia, though its diagnostic utility is controversial. The optimal criterion for $\mathrm{PaO}_{2} / \mathrm{FiO}_{2}$, respiratory index, and a-A percentage to predict 28-day mortality in our study were also found to have significant differences, as expected. We observed a considerable difference in $A B G$ predictors, including $\mathrm{PaO}_{2}, \mathrm{PaCO}_{2}, \mathrm{~A}$-a gradient, $\mathrm{AO}_{2}$, and a-A (\%) among nonsurvivors vs survivors ( $p$-value $<0.001$ ), which was expected in the disease. The hallmark of disease severity in COVID-19 patients is hypoxemia, although the associated symptoms, including respiratory distress, develop late in the disease ("silent hypoxemia"12). We calculated a criterion value of $>24.9, \leq 54$, and $\leq 7.468$ for $\mathrm{PaCO}_{2}, \mathrm{pO}_{2}$, and $\mathrm{pH}$, respectively, to predict 28-day mortality in patients developing respiratory distress and requiring NIV. Tendon et al. ${ }^{13}$ also demonstrated a significant difference in $\mathrm{PaO}_{2}$ among survivors and nonsurvivors; a statistical difference was not observed for $\mathrm{PaCO}_{2}$ and $\mathrm{pH}$.

We also compared morbidity predictors, and patient course during the ICU stay, and higher need for vasopressor requirement, continuous renal replacement therapy requirement, total parenteral nutrition requirement, and blood transfusion need among nonsurvivors. The underlying cause is multifactorial, including hypovolemia (fever, restricted fluid administration to prevent overload), vasodilation (sepsis, deep sedation during mechanical ventilation), and right or/and left ventricular dysfunction (mechanical ventilation with high positive endexpiratory pressure, pulmonary embolism, and circulating cytokines decreasing contractility, myocarditis). Although the requirement for vasopressor support is quite common in COVID19 ICU patients, the detailed hemodynamic profile or phenotype remains poorly documented. A similar duration of ICU stay was due to the development of the fibro-proliferative phase requiring oxygen supplementation, and it is further reflected by the similar need for parenteral nutrition, blood transfusion, and renal replacement therapy.

There were several limitations in this study. Although we followed a standard protocol for initiating invasive and NIV, changing guidelines from time to time concerning steroid therapy, antiviral therapy initiation, and self-proning protocol may have affected the patient outcome. Another limitation included the delayed availability of high-flow nasal cannula, which can also affect the outcome. The fact that the research was performed at 

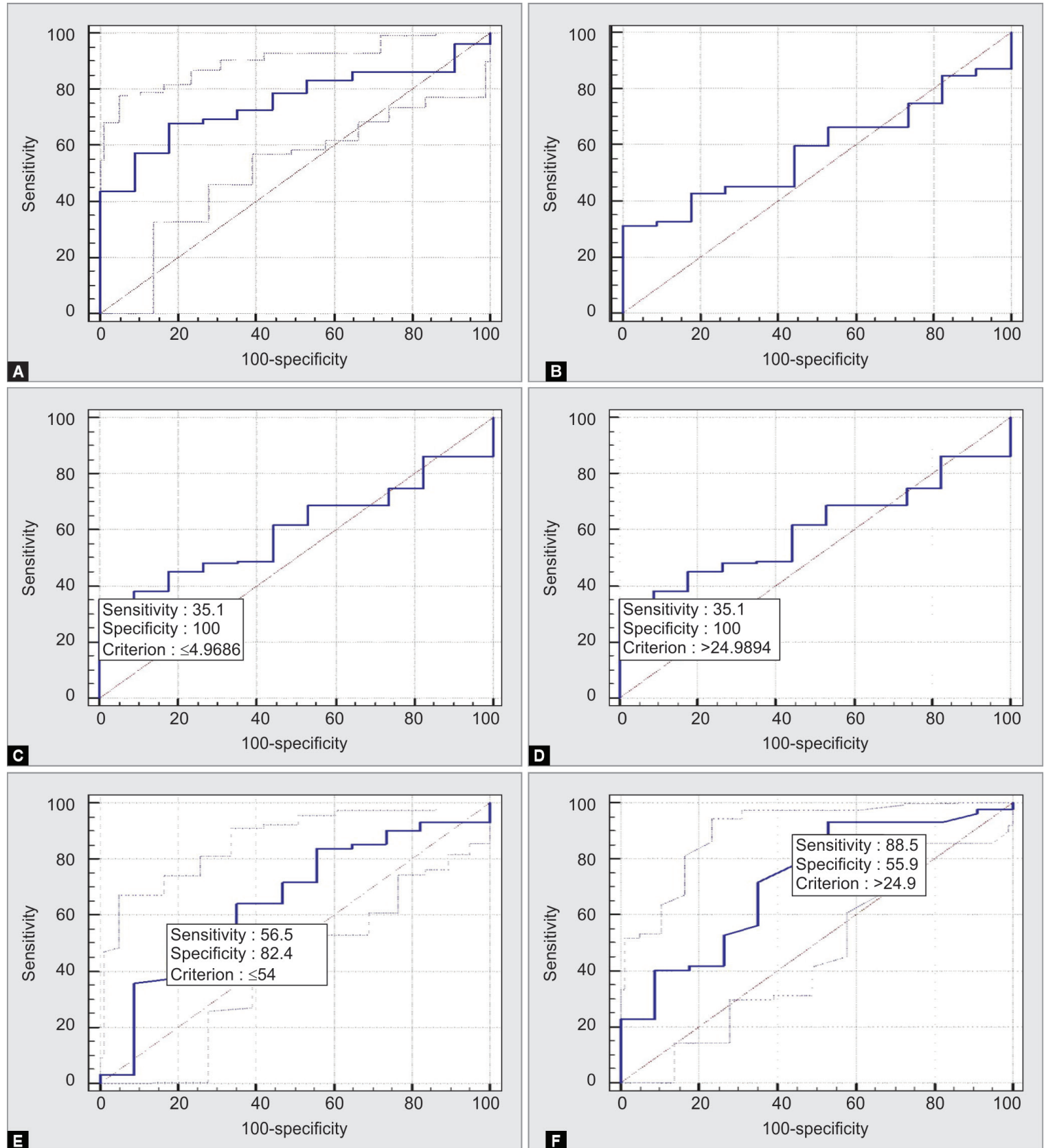

D
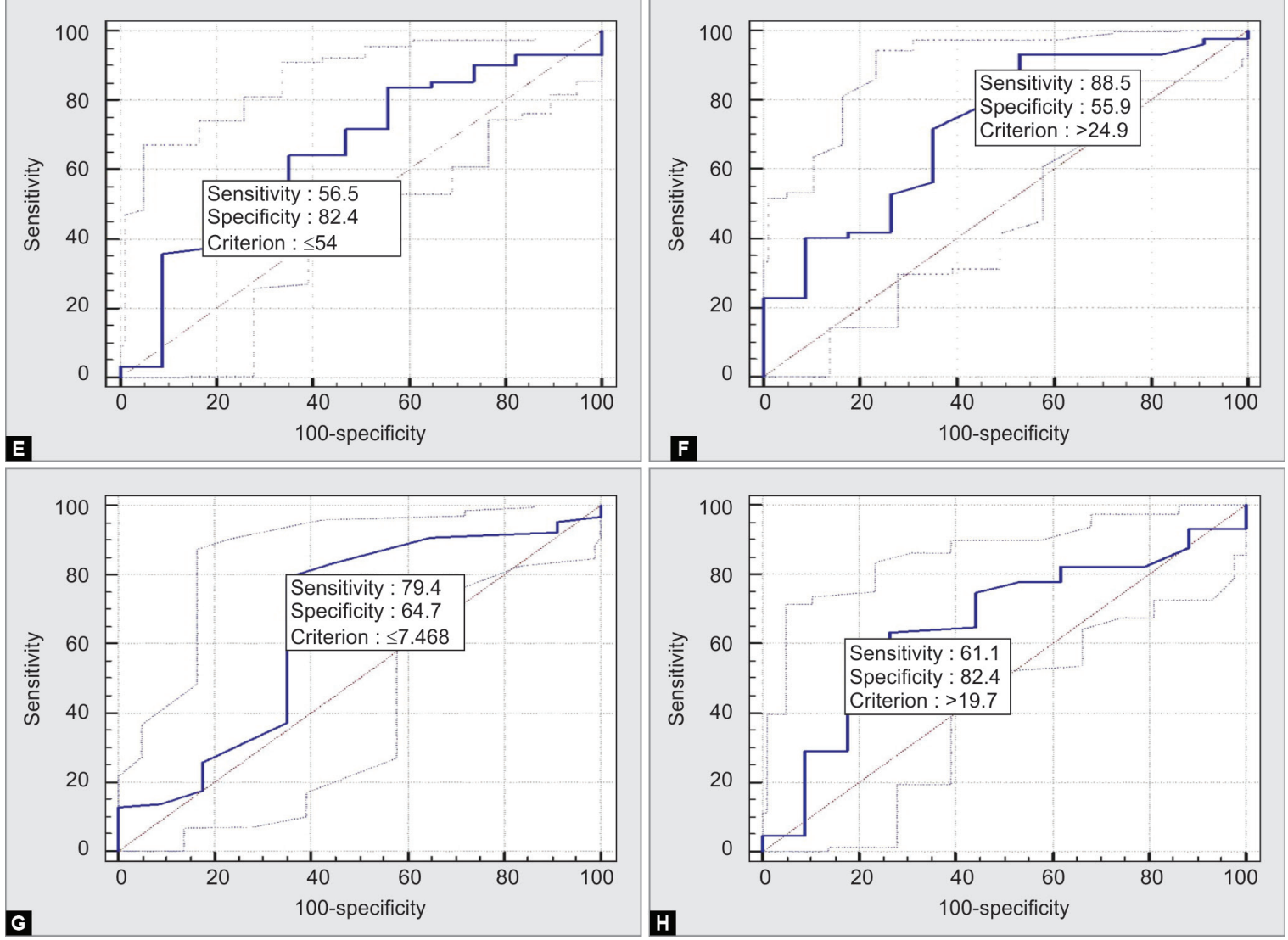

Figs $1 \mathrm{~A}$ to $\mathrm{H}$ : Receiver-operating characteristic curve for (A) a gradient; (B) $\mathrm{pO}_{2} / \mathrm{FiO}_{2} ;(\mathrm{C})$ respiratory index; (D) a-A (\%); (E) $\mathrm{PaO}_{2} ;(\mathrm{F}) \mathrm{PaCO}_{2} ;(\mathrm{G}) \mathrm{pH}$; (H) $\mathrm{HCO}_{3}$ 
one center is also a limitation of the study, restricting the findings' generalizability.

\section{CONCLUSION}

The A-a gradient is a significant predictor of mortality in patients initiated on NIV for worsening respiratory distress in COVID pneumonia. All other ABG parameters also showed a significant AUC for predicting 28-day mortality, although with variable sensitivity and specificity.

\section{HighLIGHTS}

The routine analysis of these simple, quick, readily accessible, and cost-effective $A B G$ parameters, especially the $A-$ a gradient, reliably predicted the poor prognosis of patients at NIV initiation. It will aid in the early initiation of NIV and prognostication of adverse outcomes.

\section{OrCID}

Bhavna K Gupta • https://orcid.org/0000-0002-3108-0408

Gaurav Jain 1 https://orcid.org/0000-0002-1205-7237

Saurabh Chandrakar (1) https://orcid.org/0000-0003-2449-2153

Nidhi Gupta @ https://orcid.org/0000-0001-7952-5317

Ankit Agarwal 다. https://orcid.org/0000-0003-4963-7101

\section{References}

1. Li X, Ma X. Acute respiratory failure in COVID-19: is it "typical" ARDS? Crit Care 2020;24(1):198. DOI: 10.1186/s13054-020-02911-9.

2. Armstrong RA, Kane AD, Cook TM. Outcomes from intensive care in patients with COVID-19: a systematic review and meta-analysis of observational studies Anaesthesia 2020;75(10):1340-1349. DOI: 10.1111/anae.15201.
3. Grasselli G, Greco M, Zanella A, Albano G, Antonelli M, Bellani G, et al. Risk factors associated with mortality among patients with COVID-19 in intensive care units in Lombardy, Italy. JAMA Intern Med 2020;180(10):1345-1355. DOI: 10.1001/jamainternmed.2020.3539.

4. Sarkar M, Niranjan N, Banyal PK. Mechanisms of hypoxemia. Lung India 2017;34(1):47-60. DOI: 10.4103/0970-2113.197116.

5. Dhont S, Derom E, Braeckel E, Depuydt P, Lambrecht BN. The pathophysiology of 'happy' hypoxemia in COVID-19. Respir Res 2020;21(1):198. DOI: 10.1186/s12931-020-01462-5.

6. Sharma S, Hashmi M, Burns B. Alveolar gas equation. In: Treasure Island (FL); 2020.

7. Brosnahan S, Jonkman A, Kugler M, Munger J, Kaufman D. COVID-19 and respiratory system disorders. Arterioscler Thromb Vasc Biol 2020;40(11):2586-2597. DOI: 10.1161/ATVBAHA.120.314515.

8. Burnham EL, Janssen WJ, Riches DH, Moss M, Downey GP. The fibroproliferative response in acute respiratory distress syndrome: mechanisms and clinical significance. Eur Respir J 2014;43(1):276-285. DOI: 10.1183/09031936.00196412.

9. Avci S, Perincek G. The alveolar-arterial gradient, pneumonia severity scores and inflammatory markers to predict 30-day mortality in pneumonia. Am J Emerg Med 2020;38(9):1796-1801. DOI: 10.1016/j. ajem.2020.05.048.

10. Farina G, Gianstefani A, Salvatore V, Anziati M, Baldassarri F, Beleffi M, et al. Alveolar-to-arterial oxygen gradient: role in the management of COVID-19 infection mild population. Res Sq [Internet] 2021. DOI: 10.21203/rs.3.rs-100668/v1.

11. Gilbert R, Keighley JF. The arterial-alveolar oxygen tension ratio. An index of gas exchange applicable to varying inspired oxygen concentrations. Am Rev Respir Dis 1974;109(1):142-145. DOI: 10.1164/ arrd.1974.109.1.142.

12. Xie J, Tong Z, Guan X, Du B, Qiu H. Clinical characteristics of patients who died of coronavirus disease 2019 in China. JAMA Netw Open 2020;3(4):e205619. DOI: 10.1001/jamanetworkopen.2020.5619.

13. Garciá-Tardón N, Abbes AP, Gerrits A, Slingerland RJ, Den Besten G. Laboratory parameters as predictors of mortality in COVID-19 patients on hospital admission. J Lab Med 2020;44(6):357-359. DOI: 10.1515/labmed-2020-0087. 